\title{
La industria de la información científica y el acceso al conocimiento en el siglo 21 Un modelo para el caso de Ecuador
}

\author{
Patricio Álvarez-Muñoz ${ }^{1}$, Carmen Hernández-Domínguez ${ }^{1}$ y \\ René Ramírez-Gallegos ${ }^{2}$ \\ ${ }^{1}$ Universidad Estatal de Milagro, Milagro, Guayas. Ecuador | palvarezm@unemi.edu.ec / http://orcid.org/oooo-0002-9754-8050 | \\ chernandezd@unemi.edu.ec/http://orcid.org/oooo-0oo2-2806-4917 \\ ${ }^{2}$ Centro de estudos sociais. Universidade de Coimbra. Casa Costa Alemão, Coimbra. Portugal | eltumulto@yahoo.com / https:// \\ orcid.org/oooo-0002-6837-8187
}

\section{Resumen}

Se presenta la situación de la industria de la información científica, identificando a los 5 grandes de la industria editorial. Además, se indica las diferentes formas en que los países de Latinoamérica pueden acceder a esta información. Por último, se establece un análisis comparativo de los diferentes modelos de financiamiento para acceder a la información para países en vías de desarrollo como el caso específico de Ecuador.

Palabras clave

Consorcio Ecuador Información Científica Editoriales Conocimiento

\begin{abstract}
The scientific information industry and its access to knowledge in the 21st century: A model for Ecuador. The situation of the scientific information industry is presented, identifying the 5 most important publishers. It also indicates the different ways in which Latin American countries can access this information. Finally, a comparative analysis of the different financing models for access to information for developing countries, such as the specific case of Ecuador, is established.
\end{abstract}

Artículo recibido: 16-04-2018. Aceptado: 10-08-2018
Keywords

Consortium Ecuador Scientific Information Publishers Knowledge

\section{Introducción}

La industria de la información científica, nace a partir de la formalización de la comunicación científica en los centros de conocimiento hace 350 años en Europa, y es a través de las primeras revistas científicas como la francesa Journal des Scavans y la británica Philosophical Transactions, ambas publicadas en 1665, que se consideraron los canales formales de comunicación entre científicos. 
Luego, en la década de los años 50, en EEUU esta industria se consolida, ya que es en esta década donde comienza a surgir una oferta real de información científica a través de datos a disposición de los usuarios. En el primer período, comprendido entre las décadas de los años 50 y 60 del siglo XX, el nacimiento de esta gran industria se conoce gracias a los estudios realizados por Hartley; Keen; Large y Tedd (1990), Lancaster y Warner (1993) y Neufeld y Cornog (1986); en un segundo periodo, en la década de los 70, a través de los trabajos de Bjørner y Ardito $(2003,2004)$ y Hahn (1996).

En esta industria, el bien estrella que se comercia es un bien público (Stiglitz y Greenwald, 2014), pero que por la influencia de las fuerzas de mercado, ha resultado ser susceptible a una mercantilización. Así en países como EEUU o de la Unión Europea, los niveles de mercantilización de la información científica a través de los modelos de negocios implementados por esta industria llegan a ser inmanejables para las universidades públicas y centros de investigación (Owens, 2012).

Un caso especial es el de América Latina, ya que tiende a ser muy diferente al esquema anglosajón. De hecho, muchos países como México, Colombia, Brasil, Ecuador y Bolivia, entre otros, consideran conceptualmente el conocimiento como un bien de acceso universal. Como toda industria con características oligopólicas, detrás de este esquema de mercado existen grandes conglomerados empresariales que lo estructuran. Es el caso de esta industria, donde no son muy visibles los holdings sino las editoriales a las que ellos pertenecen.

Con esta aportación se ayudará a identificar los grandes grupos empresariales en los que se asienta toda esta industria y como influyen en el mundo académico a través del sistema de comunicación científica. Es necesario saber que el proceso de la comunicación científica, tal y como se conoce en la actualidad, funciona mediante un proceso de envío de un artículo científico a una revista, que es sometido a un proceso de evaluación por pares ciegos, quienes deciden si el documento es o no publicable. De ser positiva la evacuación, la revista notifica al autor, quien pagará y cederá los derechos de autor para realizar la distribución y publicación en las distintas revistas científicas. Las revistas, a su vez, son propiedad de editoriales privadas, y son presentadas ante la comunidad a través de las bases de datos de referencia, que son propiedad en algunos casos de estas grandes editoriales.

De esta manera se puede comprender lo que Brian Nosek, profesor de la Universidad de Virginia y director del Centro para la Ciencia Abierta, manifiesta a través del trabajo de Schmitt: "La publicación académica es el modelo de financiación perfecto para hacer un montón de dinero, ya que el producto y el consumidor son la misma persona" (Schmitt, 2015).

Dicho esto, en el mercado se identifican tres grandes holdings como las plataformas proveedoras más grandes de información científica en el mundo. En base a los ingresos y participaciones en el mercado que tienen estos holdings, se puede decir que ejercen un poder de mercado como nunca antes se ha visto en un determinado sector económico. Estos holdings son Reed Elsevier, Clarevite (antes Thompson Reuters), y recientemente, Google. Son considerados los más importantes ya que el mundo académico percibe que la ciencia no avanzaría en términos de calidad si estas revistas no aplicaran el proceso de revisión por pares, que garantiza y valida la calidad de los contenidos (Romanos de Tiratel, 2008).

En concreto, el modelo de financiación actual de esta industria es el pago por uso o pay per use. Con todo, desde el punto de vista de la demanda, esto es, de autores, bibliotecas y gobiernos, y su constante necesidad de información científica, provoca 
que la tendencia creciente sea la creación de consorcios. Esta situación se hace más profunda debido a que el número de investigadores está en constante aumento, y su volumen de producción es cuantitativamente superior al número de revistas disponibles, lo que crea una brecha de la que se aprovechan las mismas editoriales. Esto es, hablando en términos económicos: la demanda supera a la oferta.

Si se comparan los beneficios obtenidos por las compañías de cada industria, se obtiene que Elsevier y Springer concentran mayores porcentajes de beneficios que muchas de las compañías del sector industrial, automovilístico, editorial y minero. Según Larivière, Haustein y Mongeon (2015) se puede generar la siguiente tabla:

\begin{tabular}{llc}
\hline Compañía & Industria & $\begin{array}{c}\text { Beneficio } \\
\%\end{array}$ \\
\hline Elsevier & Publicación Académica & 37 \\
Springer & Publicación Académica & 35 \\
Apple & Computación & 29 \\
Google & Busqueda de información & 25 \\
Rio Tinto & Mineria & 23 \\
BMW & Automoviles & 10 \\
\hline
\end{tabular}

Elaborado por el autor a partir de Larivière, Haustein y Mongeon (2015)

Con respecto a las cuotas de mercado de las casas editoras, Elsevier concentra el 13,5\% de la cuota de mercado, frente al 8,3 de Springer y el 6,0\% de Wiley.

Por último, cuando se habla de la comercialización de los productos de dichas editoriales, se muestra que estas mantienen todo un portafolio de acuerdo con las necesidades del mercado.

\section{Metodología de análisis}

Térmens-Gruells (2007) y Tenopir (2003) proponen combinar las metodologías cuantitativa y cualitativa como el proceso idóneo en las investigaciones en documentación e información. En este caso se ha decidido emplear el método cuantitativo, método demostrado eficaz, porque de esta manera, al desarrollar investigaciones enfocadas a Ecuador, se ayudará a abrir una línea de investigación en documentación e información pionera en el país, potenciando estudios ya existes en Latinoamérica. Pudiéndose complementar con investigaciones guiadas por metodologías cualitativas.

En este sentido, el enfoque cuantitativo queda ampliamente integrado, por ejemplo, en la aplicación de la estadística inferencial y descriptiva y en el análisis de las externalidades económicas.

Para describir el estado actual de la industria, recurrimos a la metodología documental: se barren las bases de datos de información científica más importantes del mundo, Scopus y Web of Science, para recolectar información actualizada sobre la industria. También recolectamos información de las propias páginas web oficiales de cada editorial. Luego, aplicando métodos cuantitativos, como la estadística descriptiva, se tratan los datos recolectados y se presentan a través de figuras y tablas que sirven para explicar de mejor manera la situación actual de la industria científica.
Tabla 1. Comparación de Beneficios por compañía en el año 2015 
Figura 1. Comparación de ingresos de las cinco editoriales analizadas en Reino Unido

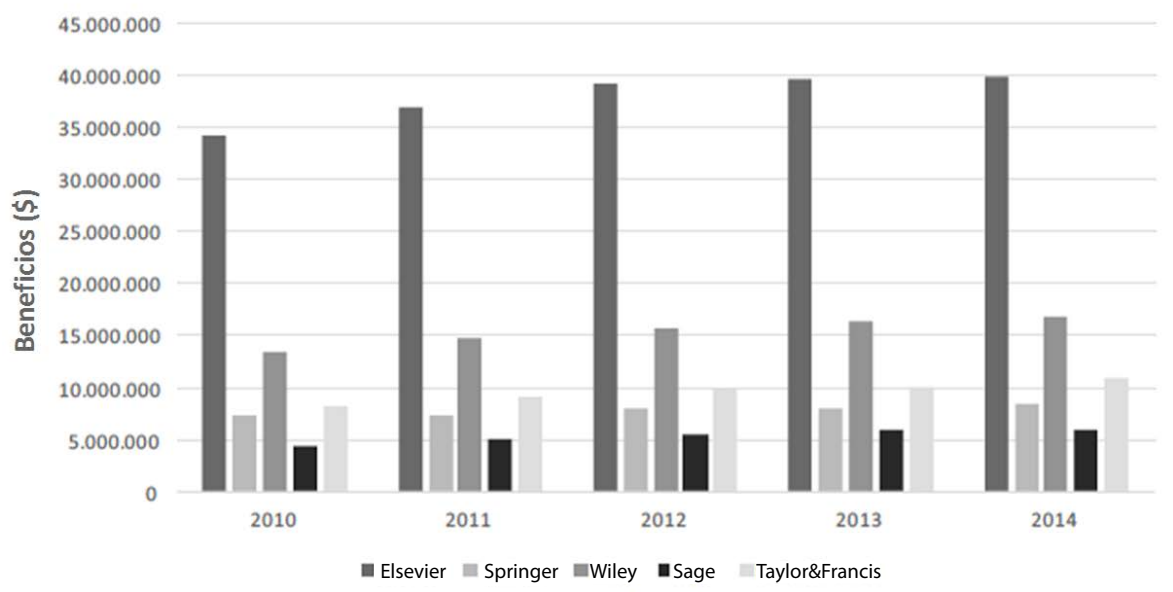

\section{Resultados}

La constante dificultad de acceso a la información y al conocimiento que tienen los académicos, investigadores y estudiantes, provocada por el modelo de financiación implantado por las grandes editoriales, es una circunstancia que cada día se hace más evidente por los problemas económicos que atraviesan las instituciones universitarias. Consecuencia del modelo de financiación imperante, y sumado a las políticas financieras de las editoriales, regiones enteras terminan perjudicadas, especialmente regiones donde existen países en vías de desarrollo.

No es de extrañar, que el acceso a la mayoría de las revistas es prohibitivamente caro para cualquier persona ajena al mundo académico. Incluso si el actual movimiento de acceso abierto tiene más éxito, la jerga incomprensible y el mero volumen y longitud de los documentos seguirían impidiendo a los profesionales leerlos.

En todo este contexto, existen cinco grandes grupos editoriales, a saber: Elsevier, Springer, Wiley, TaylorEFrancis y Sage, que controlan más del 50\% de los artículos publicados. Este mercado está liderado por Elsevier, que reportó un margen de beneficios del 37\%, y con ingresos de 3,5 mil millones de dólares. Estos beneficios e ingresos son más altos que los de Apple Inc. en el año 2014. Como manifiesta Watt (2016), esos beneficios son producto casi en su totalidad del cobro a los investigadores.

En concreto el sistema no es útil a las necesidades de la comunidad científica debido a cuatro factores (Eisen y Volsshall, 2016):

1. "Es lento. Los manuscritos pasan una media de nueve meses en revisión por pares antes de la publicación, y los árbitros demandan en forma creciente cada vez más datos y más experimentos para endosar un artículo para publicación. Estas demoras enlentecen de manera masiva la difusión del conocimiento."

2. "Es caro. Se gastan \$10 mil millones al año en la publicación de revistas en ciencia y medicina, más de \$6.000 por artículo, y en forma creciente estos costos provienen de las becas de investigación."

3. "Es arbitrario. El sistema actual de revisión por pares es defectuoso. Artículos excelentes son rechazados, y se aceptan artículos defectuosos. A pesar de esto, el nombre de la revista continúa siendo como un indicador de calidad del artículo".

4. "Es inaccesible. Incluso con los esfuerzos significativos del movimiento del acceso abierto la vasta mayoría de la literatura científica no es accesible sin una suscripción". 
Todo esto deriva en un bajo impacto social de las publicaciones, trastocándose el fin de la investigación porque es más importante publicar que el impacto que puede o debe tener la investigación en la sociedad.

Para los investigadores de las universidades más pequeñas en el mundo, los costos de acceso a la información científica son casi prohibitivos. Esto deja a los académicos, investigadores y estudiantes en la completa oscuridad cognitiva (Watt, 2016).

El esquema creado por las editoriales obliga a que la revista publique el artículo a través de un paywall ${ }^{1}$ o muro de pago, que tiene un costo para su acceso de alrededor de 25 dólares por artículo (Watt, 2016).

Como consecuencia, en la actualidad, las editoriales comerciales no se inclinan tanto al cobro por artículo, sino que han creado varios mecanismos para que las bibliotecas universitarias accedan a la información que ellos contienen. Entre otros, por ejemplo, crear paquetes de revistas con el solo fin de, aparentemente, aliviar la carga financiera a las bibliotecas universitarias. Estos paquetes de revistas están compuestos por una mezcla de revistas de alta calidad y revistas de baja calidad. Aunque hay que aclarar que, en el caso de la editorial Elsevier, con un inventario de cerca de 2.500 revistas, la mayoría de estas son de la misma calidad (Watt, 2016).

Este sistema, con sus altos costos, es la preocupación del mundo académico, ya que el progreso científico se basa, precisamente, en el acceso a los descubrimientos de sus predecesores. Cuando se niega este acceso a través de estos modelos de financiación, la tasa de progreso de la ciencia, por lo general, se ve revertida.

\section{Modelos}

En la dinámica del mundo académico se identifican 3 modelos de financiación y acceso a la información científica (Dosdoce, 2015), estos son:

» Modelo clásico

» Modelo Open Access

» Modelos de financiación surgidos de la irrupción de Internet

- Micropagos-contenido fraccionado

- Pago por consumo: streaming y pay per view

- Modelos de suscripción

- Membresía

- Freemium-Premium

- Por publicidad insertada

- P2P-Mooca

El modelo clásico que utilizan las editoriales para distribuir sus productos está basado en conseguir la mayor rentabilidad posible. La industria de las publicaciones científicas bajo este modelo clásico de suscripción genera entre 5 y 6 mil millones de dólares de beneficios por publicar 2 millones de artículos aproximadamente (Peláez, 2016).

El modelo de financiación tradicional, de suscripciones, es aquel en el que los costes recaen en los suscriptores, quienes a través de sus cuotas cubren los gastos editoriales y generan beneficios a los editores (Cabezas, 2007). Adicionalmente, en este modelo ni autores ni revisores cobran por el trabajo realizado.

Con este esquema, países como España, a través de sus universidades públicas y el CSIC (Centro Superior de Investigaciones Científicas) pagan algo más de 115 millones
1. El Paywall es un sistema que impide que los usuarios de internet tengan acceso a determinadas páginas web (noticias y publicaciones académicas) 
2. La Declaración de Bethesda sobre Publicación de Acceso Abierto (Scalable Processor ARChitecture, 2003), la Declaración de Budapest (BOAI, 2016) y la Declaración de Berlín (Sociedad Max Planck, 2003), desarrollada por el Instituto Max Planck.
3. También existen otras formas de adquirir recursos de información científica que no están dentro del esquema de un modelo de financiación, ya que son casos puntuales. A esto se lo conoce como grupos de compras, se dan generalmente en hospitales, asociaciones profesionales; $y$ en España se sabe que intervienen en universidades como la de La Laguna, Complutense, Universidad de Valencia, Universidades de Castilla y León, que no obtienen accesos a recursos de información científica mediante consorcios. de euros anuales para tener acceso a estas publicaciones científicas (CRUE, 2016). De la misma manera, el gobierno de Francia, a través de su ministerio de investigación pagó 172 millones de euros al líder mundial de publicaciones científicas, Elsevier (France prefers, 2014).

En los últimos años, surgió el movimiento Open Access como respuesta al modelo clásico, que sigue imperante en el mundo. Con las declaraciones a favor del acceso abierto, conocidas como las tres $\mathrm{B},{ }^{2}$ se estableció el punto de partida para que este modelo sea considerado hoy una alternativa consistente. En la actualidad, existen declaraciones a favor del Open Access, como la Declaración de Dakar sobre publicaciones de acceso abierto del 1 de abril de 2016 (CODESRIA, 2016); y la declaración conjunta COAR-UNESCO sobre acceso abierto, celebrada el 9 de mayo de 2016 (COAR-UNESCO, 2016).

El modelo Open Access consiste en que el lector no tiene que pagar por acceder a los artículos, ya que los gastos editoriales y los posibles beneficios son cubiertos por el autor del artículo o por la institución en que este trabaja (vía dorada). Así también existe el modelo por el que se accede a la información a través de los repositorios institucionales (vía verde).

Una de las características de las revistas en acceso abierto es que alcanzan una gran difusión, consiguiendo en muchos casos mayor visibilidad del autor por sus investigaciones. De esta manera, esta tendencia se está imponiendo en los últimos años y se ve fortalecida por la irrupción y el desarrollo sin precedentes de Internet, que ha propiciado que las publicaciones científicas estén accesibles al público en general.

En una discusión, celebrada en Reino Unido en el año 2010, acerca de las características de los mercados de revistas científicas se tomaron en consideración tres criterios para poder comparar el nuevo mercado de Open Access con el mercado clásico de suscripciones: la concentración de mercado, las barreras de entrada y la capacidad de respuesta de los clientes (Jisc, 2016). En la tabla 2 podemos ver resumidas las principales ideas resultantes de esa comparación ${ }^{3}$.

A su vez, en la economía política de la difusión del conocimiento es necesario tomar en cuenta dos fenómenos adicionales: por una parte, el crecimiento de revistas predatorias privadas de baja calidad que constituyen fraudes académicos y científicos (Kolata, 2017); y, por otro, la "tiranía de las revistas de lujo que cada vez más imponen qué se publica en función de los temas científicos de moda, de criterios editoriales y de la reducción artificial del número de trabajos que aceptan para subir su demanda y prestigio", tal cual fue denunciado por el Premio Nobel de medicina Randy Schekman al diario The Guardian (Schekman, 2013).

\section{América Latina y Ecuador}

En este escenario, surgen actores como el Gobierno, las universidades y las instituciones para implementar acciones como consorcios, acuerdos puntuales o iniciativas individuales para poder sentarse en la mesa de negociaciones con las grandes editoriales, como los consorcios que ya existen en Chile y Perú. Así, esta participación y dinámica permite enfrentar dificultades, técnicas, operativas y financieras que han perjudicado la posibilidad de mantener el acceso a los recursos de información científica en las universidades de América Latina. 


\begin{tabular}{ll}
\hline Criterio & Nuevo Mercado Open Access \\
\hline $\begin{array}{l}\text { Concentración de } \\
\text { mercado }\end{array}$ & Mercado con baja concentración. En 2015, \\
& solo el 16\% de todos los títulos de revistas \\
& en OA fueron publicados por los 10 editores \\
& con más títulos
\end{tabular}

Mercado clásico de suscripciones

La concentración de marcado es relativamente grande En 2009, el $35 \%$ de los títulos de las revistas se publicaron por las 19 mayores editoras, pero sólo en 5 años esa cifra aumentó al $45 \%$, principalmente debido a que las grandes casas comerciales continúan haciéndose cargo de las operaciones editoriales de sociedades científicas, y que operan cerca de las comunidades académicas

\begin{tabular}{|c|c|c|}
\hline Barreras de entrada & $\begin{array}{l}\text { Hay bajas barreras de entrada. } \\
7 \text { de los } 10 \text { editores de revistas OA con la } \\
\text { mayor gama de títulos fueron fundados } \\
\text { desde } 2010 \text {. } \\
\text { El éxito de los nuevos editores en el mercado } \\
\text { del OA (PLOS y Hindawi) es evidencia de que } \\
\text { estos mercados cuentan con altos niveles de } \\
\text { innovación, nuevas tecnologías y modelos } \\
\text { de negocio, que permiten a los nuevos } \\
\text { operadores operar a escala y competir con } \\
\text { los operadores tradicionales }\end{array}$ & $\begin{array}{l}\text { Hay altas barreras de entrada. } 8 \text { de los } 10 \\
\text { editores de revistas con la mayor gama de } \\
\text { títulos fueron fundadas antes de } 1900 \\
\text { Una variedad de características } \\
\text { estructurales limita las oportunidades para } \\
\text { los nuevos participantes, y crean barreras } \\
\text { importantes que son muy pocos los nuevos } \\
\text { editores que han sido capaces de superar } \\
\text { en los últimos años }\end{array}$ \\
\hline $\begin{array}{l}\text { Capacidad de } \\
\text { respuesta de los } \\
\text { clientes }\end{array}$ & $\begin{array}{l}\text { La respuesta de los clientes en términos de } \\
\text { sensibilidad a los precios es relativamente } \\
\text { fuerte. } \\
\text { “...creemos que para revistas OA completas, } \\
\text { la sensibilidad de los autores a los niveles de } \\
\text { APCs ha estado trabajando efectivamente en } \\
\text { crear presión para moderar el precio de los } \\
\text { APCs.” }\end{array}$ & $\begin{array}{l}\text { La respuesta de los clientes en términos de } \\
\text { sensibilidad a los precios es débil. } \\
\text { “...los artículos híbridos de OA son } \\
\text { significativamente más caros que sus } \\
\text { homólogos completos de OA y el nivel } \\
\text { de precios es un factor importante en la } \\
\text { inhibición de la adopción de la opción } \\
\text { híbrida” (Bjork \& Solomon, 2014). }\end{array}$ \\
\hline
\end{tabular}

En este sentido, en Latinoamérica se están empleando tres modelos que permiten la adquisición de licencias para el acceso a los recursos electrónicos de información científica. Esto explica en parte el crecimiento visible de las publicaciones científicas indexadas en las grandes bases de datos de referencia, como WoS (Clarevite) y Scopus (Elsevier), ya que, al disponer del acceso a estas bases de datos, permitirá conocer más revistas especializadas y estados del arte actualizados que facilitan la generación de más conocimiento. Esta circunstancia ha provocado que estas compañías comiencen a tomar en cuenta la creciente demanda de información científica que se está generando en esta región, como es la estrategia de ampliación geográfica de Scopus al indexar más revistas latinoamericanas, y la de WoS al adquirir Scielo. 
Tabla 3. Ránking de los 10 países con mayor producción científica en América Latina y el Caribe (base de datos Scopus), 1996-2006-2016

\begin{tabular}{|l|r|}
\hline \multirow{2}{*}{ País } & \multicolumn{1}{|c|}{ Año } \\
\cline { 2 - 2 } Brazil & \multicolumn{1}{c|}{1996} \\
\hline Mexico & 8.814 \\
\hline Argentina & 4.593 \\
\hline Chile & 4.093 \\
\hline Venezuela & 1.005 \\
\hline Cuba & 744 \\
\hline Colombia & 572 \\
\hline Puerto Rico & 457 \\
\hline Uruguay & 263 \\
\hline Costa Rica & 259 \\
\hline
\end{tabular}

\begin{tabular}{|l|r|}
\hline \multirow{2}{*}{ País } & \multicolumn{1}{|c|}{ Año } \\
\cline { 2 - 2 } Brazil & \multicolumn{1}{c|}{$\mathbf{2 0 0 6}$} \\
\hline Mexico & 33.816 \\
\hline Argentina & 12.309 \\
\hline Chile & 7.703 \\
\hline Colombia & 4.954 \\
\hline Venezuela & 1.093 \\
\hline Cuba & 1.804 \\
\hline Puerto Rico & 919 \\
\hline Peru & 656 \\
\hline Uruguay & 627 \\
\hline
\end{tabular}

\begin{tabular}{|l|r|}
\hline \multirow{2}{*}{ País } & \multicolumn{1}{|c|}{ Año } \\
\cline { 2 - 2 } Brazil & \multicolumn{1}{|c|}{$\mathbf{2 0 1 6}$} \\
\hline Mexico & 68.908 \\
\hline Argentina & 12.864 \\
\hline Chile & 12.448 \\
\hline Colombia & 9.481 \\
\hline Ecuador & 2.249 \\
\hline Peru & 2.228 \\
\hline Cuba & 1.695 \\
\hline Venezuela & 1.476 \\
\hline Uruguay & 1.472 \\
\hline
\end{tabular}

Fuente: Scopus. Elaboración: propia

Analizando los datos recogidos en la Tabla 3, se observa que Colombia es un caso emblemático, porque es uno de los países en la región que más crece en generación de conocimiento, viéndose reflejada en sus publicaciones científicas en revistas de impacto. COLCIENCIAS tiene como misión "liderar, orientar y coordinar la política nacional de ciencia, tecnología e innovación, y el Sistema Nacional de Ciencia, Tecnología e Innovación para generar e integrar el conocimiento al desarrollo social, económico, cultural y territorial del país" (COLCIENCIAS, 2015). Este consorcio maneja un modelo de adquisición de recursos de información científica de tipo centralizado. Asimismo, mantiene un acuerdo con la empresa editorial Elsevier, gracias al cual se ofrece a la comunidad científica colombiana el acceso a recursos de información científica. Los beneficiarios de este acuerdo son 38 centros de investigación y 26 universidades públicas y privadas (COLCIENCIAS, 2015), los cuales pueden acceder a los siguientes productos: ScienceDirect, Compendex, Reaxys, Embase y Scopus. En la tabla 3 se presenta en ránking de los países latinoamericanos con mayor producción científica.

México también sobresale en cuanto a generación de conocimiento y por la forma de acceder a la información científica. Existe el consorcio CONRICYT, formado en el año 2009 como una iniciativa de la Secretaría de Educación Pública, el Consejo Nacional de Ciencia y Tecnología (CONACYT), la Asociación Nacional de Universidades y Educación Superior, la Universidad Autónoma Metropolitana, la Universidad Autónoma de México, el Instituto Politécnico Nacional, el Centro de Investigación y Estudios Avanzados del Instituto Politécnico Nacional, la Universidad de Guadalajara y la Corporación Universitaria para el Desarrollo de Internet. Este consorcio tiene como principal objetivo ampliar y volver más ágil el acceso a la información científica (bases de datos y revistas electrónicas reconocidas en circuito científico) (CONACYT, 2015).

De la misma manera, Chile tiene el consorcio CINCEL (Consorcio para el Acceso a la Información Científica-Electrónica), que fue creado en 2002. Este consorcio está constituido por 25 universidades, la Comisión Nacional de Investigación Científica y Tecnológica y el CONICyT. En el 2007 se integra el Instituto Antártico Chileno. En 2011 se asocia el Servicio Nacional de Geología y Minería. Y, finalmente, en 2013 se asocia al Ministerio de Educación.

Se puede argumentar que los países latinoamericanos que en la actualidad tienen visibilidad científica a través de sus artículos científicos mantienen, en su mayoría, 
modelos de adquisición de recursos de información científica a través del modelo de consorcio, quienes muchas veces reciben aportes gubernamentales.

Ecuador, tuvo un proceso de reforma de la educación superior en donde uno de los cambios institucionales importantes fue la articulación en una misma cartera de Estado la Educación Superior con la Ciencia, Tecnología, Innovación y saberes ancestrales (SENESCYT). La reforma tuvo como uno de sus objetivos el fomento a la producción científica. Entre 2006 y 2016, Ecuador, fue el país que más creció en América Latina y el Caribe en publicar en revistas indexadas en SCOPUS y en términos absolutos pudo entrar en el ranking de los 10 países con mayor nivel de publicaciones de la región. Antes de la reforma, muy pocas universidades contaban con bases de datos científicas. Como parte de la política pública para crear la cultura de la investigación, en un principio, la política pública fue financiar a todas las universidades e institutos de investigación pública. A medida que se generaba la cultura de la demanda de información científica, el financiamiento se iría trasladando a las instituciones. Luego de 5 años, la SENESCYT implementó un modelo de cofinanciamiento o copago, con un esquema del 50-50. Esto es, el $50 \%$ del valor de las bases de datos seleccionadas lo financia la universidad pública y el 50\% restante el Estado a través de la SENESCYT. Este acuerdo tenía un periodo de vigencia de un año, según acordaran ambas partes. Posteriormente las universidades debían abonar anualmente el monto de su suscripción de acuerdo con las necesidades de la institución. En el año 2015 el modelo dejó de funcionar, dejando a las universidades públicas y privadas la conducción del tema de manera individual.

Según se refleja en la tabla 4, la inversión destinada por el Gobierno a la adquisición de accesos a recursos electrónicos fue incrementándose año a año. Mientras el modelo se mantuvo vigente, el periodo comprendido entre los años 2012 y 2014 fue en el que más inversión se realizó, en un promedio de más del $232 \%$.

\begin{tabular}{|c|r|}
\hline Año & Inversión (\$) \\
\hline 2010 & 1.983 .000 \\
\hline 2011 & 2.440 .000 \\
\hline 2012 & 2.600 .000 \\
\hline 2013 & $5.214 .605,55$ \\
\hline 2014 & $8.652 .898,34$ \\
\hline
\end{tabular}

Tabla 4. Inversión del gobierno en bases de datos de información científica

\section{Discusión}

Los costos para poder acceder a las publicaciones científicas no se deben solamente a las elevadas cantidades que cobran las editoriales, sino también a la forma en que estas venden sus productos. En concreto, una biblioteca no puede suscribirse a las revistas que realmente necesita, sino que tiene que comprar "paquetes". Estos paquetes, además de contener las revistas que necesita la biblioteca, incluyen otras que no son de su interés.

En los últimos años, ante las situaciones como la irrupción del Open Access y la dificultad de las bibliotecas en la negociación de las suscripciones ha provocado que las editoriales más grandes del mundo no se hayan quedado de brazos cruzados ante estas situaciones. Así, la editorial Wiley, de John Wiley ESons, decidió poner en marcha el modelo Open Access mediante la implementación del Wiley Open Access (Wiley, 2016). A su vez, editoriales como Springer, Wolters Kluwer, Flat World Knowledge o Elsevier también están adoptando una opción de acceso abierto para sus publicaciones (Martín Gavilán, 2009). 


\begin{tabular}{|c|c|c|}
\hline Modelo de negocio & Fortalezas & Debilidades \\
\hline Consorciado & $\begin{array}{l}\text { - Fomento a la cooperación entre los } \\
\text { miembros } \\
\text { - Mayores posibilidades de negociación } \\
\text { - Incremento de la información ofrecida a } \\
\text { los usuarios } \\
\text { - Estabilidad en los acuerdos } \\
\text { - Disminución de los gastos de negociación } \\
\text { y gestión } \\
\text { - Disminución de los costos generados en } \\
\text { las adquisiciones }\end{array}$ & $\begin{array}{l}\text { - Dificultad para llegar a acuerdos } \\
\text { internos } \\
\text { - Diferente velocidad de desarrollo } \\
\text { entre consorcio y sistema } \\
\text { bibliotecario }\end{array}$ \\
\hline Gobierno único proveedor & $\begin{array}{l}\text { - Aparición de monopsonios } \\
\text { - Mayor poder de negociación }\end{array}$ & $\begin{array}{l}\text { - Burocratización de los procesos } \\
\text { - Profundo desconocimiento de las } \\
\text { dinámicas del sistema bibliotecario } \\
\text { (muchas veces los grupos } \\
\text { negociadores están formados por } \\
\text { personas que desconocen al ámbito } \\
\text { bibliotecario) } \\
\text { - Realidad distorsionada de las } \\
\text { necesidades de las universidades } \\
\text { públicas } \\
\text { - Dependencia directa del } \\
\text { presupuesto público }\end{array}$ \\
\hline Individual & $\begin{array}{l}\text { - Libertad al escoger productos } \\
\text { - Libertad de negociación }\end{array}$ & $\begin{array}{l}\text { - Limitaciones presupuestarias } \\
\text { - Menor poder de negociación } \\
\text { - Limitaciones para crear economías } \\
\text { de escala }\end{array}$ \\
\hline Pay per use & $\begin{array}{l}\text { - Racionalización del gasto } \\
\text { - Se adquiere lo que es realmente necesario }\end{array}$ & $\begin{array}{l}\text { - Costo unitario superior a costo de } \\
\text { paquete } \\
\text { - Modelo restringido por parte de las } \\
\text { compañías } \\
\text { - Desconocimiento de la tasa de } \\
\text { descargas }\end{array}$ \\
\hline
\end{tabular}

Tabla 5. Matriz comparativa de los modelos ajustada al contexto ecuatoriano

La dinámica de este mercado se refleja en que los científicos pagan entre 3 y 5 mil dólares para publicar sus descubrimientos en revistas como Science, Cell o Nature. Si sus trabajos responden a los requisitos del mercado -más allá de la calidad de sus intervenciones-, luego acuerdan un contrato que los compromete a ceder sus derechos y les impide compartir el contenido por intermedio de cualquier otro medio (Esteban, 2018).

En países como Ecuador, donde se ha conseguido que el sistema de educación superior mejore cuantitativa y cualitativamente, se debe adoptar un modelo que permita establecer un ecosistema para la investigación y esta a su vez se nutra de la información científica actualizada que se genera alrededor del mundo. Así el modelo de compras consorciadas favorece la creación de consorcios bibliotecarios en el sistema universitario y permite que las bondades del modelo generen acciones coordinadas (Anglada i de Ferrer, 2003). La propuesta señalada es acorde también a la mirada de la reforma planteada en Ecuador en donde el núcleo central es generar redes de conocimiento; es decir, la estrategia de circulación de la información científica debería estar articulada a que en la difusión del conocimiento se parte de una apuesta más ambiciosa: la creación de una cultura de trabajo 
colaborativo en red para la producción científica, no solo al interior del país sino también con horizontes trans-estatales.

Las bibliotecas universitarias, al igual que los entes vivos, se desarrollan por su capacidad de cooperar (Anglada i de Ferrer, 2003). Desde el punto de vista metodológico, existen varias investigaciones que ayudan a comprender la actividad de los consorcios: Potter (1997); Kopp (1998); McFadden Allen y Hirshon (1998); e IFLA (2001).

¿Cuáles son las ventajas y desventajas entre un modelo de consorcio frente a "Pay per Use", "Gobierno único negociador y proveedor", "Modelo individual"? Analizando la tabla 5 vemos las diferentes características y bondades de cada modelo.

Desde un enfoque institucional y partiendo del hecho que -hoy en día- es inevitable dejar de comprar las bases científicas que permitan el acceso al conocimiento, de acuerdo con la matriz, el modelo consorciado es el modelo que más ventajas reales presentaría para el contexto de las universidades. Se observa que todos los modelos, en algún aspecto, dependen de fondos públicos, por lo que el modelo consorciado es el que mejor aprovecha la asignación de estos fondos ya que es el único que crea economías de escala4.

\section{Conclusiones}

Los grandes conglomerados empresariales están empeñados en consolidar su posición y derivan todos sus esfuerzos en evitar que existan nichos periféricos regionales y así consolidar el monopolio de la distribución de la ciencia en el mundo.

América Latina se encuentra inmersa en un proceso de crecimiento en la generación de conocimiento (Álvarez-Muñoz y Pérez-Montoro, 2015). Países andinos como Colombia, Ecuador, Perú y Chile se están tomando muy en serio el tema de la visibilización científica a través de revistas indexadas en las bases de datos de referencia bibliográficas más importantes del mundo.

Para Ecuador, el implementar el modelo de consorcios para el acceso a la información científica es la vía mas recomendable tomando en consideración la situación política y económica que atraviesa el país. Esta propuesta puede ser replicada también a cualquier país en vías de desarrollo.
4. En la II Reunión Iberoamericana de Ministros y altas autoridades de Ciencia, Tecnología e Innovación de la región, celebrada en Cartagena de Indias-Colombia en octubre del 2016, se propuso negociar la compra de bases de datos como bloque de gobierno. En el marco de UNASUR o CELAC, esta agenda podría ser estratégica que viabilizaría mayor difusión y ahorro por país. Es necesario hacer un análisis riguroso de ventajas y desventajas tanto cualitativas como económicas de la generación de un consorcio de gobiernos que compren las bases de datos para difundir en todas las instituciones que generan conocimiento de los países de la región. 


\section{Q Referencias bibliográficas}

"Álvarez-Muñoz, Patricio y Mario Pérez-Montoro. 2015. Análisis de la producción y visibilidad de la producción científica de Ecuador en el contexto andino (2000-2013). En El profesional de la información. Vol. 24, no. 5, 577-586. https:// doi.org/10.3145/epi.2015.sep.07

»Anglada i de Ferrer, Lluís M. 2003. Impacto e influencia de los consorcios en la gestión de colecciones. En BiD. No. 10. <http://bid.ub.edu/1oangladaz.htm> [Consulta: 17 enero 2016].

»Bjørner, Susanne y Stephanie C. Ardito. 2003. Online before the internet, Part 1: Early pioneers tell their stories. En Searcher. Vol. 11, no. 6, 36. <http://www.infotoday.com/searcher/juno3/ardito_bjorner.shtml> [Consulta: 17 enero 2016].

» Bjørner, Susanne y Stephanie C. Ardito. 2004. Online Before the Internet, Part 5: Early Pioneers Tell Their Stories. En Searcher. Vol. 12, no. 1, 40-49. <http://www. infotoday.com/searcher/jano4/ardito_bjorner.shtml> [Consulta: 17 enero 2016].

» BOAI. 2016. Budapest Open Access Initiative. Iniciativa de Budapest para el Acceso Abierto. <http://www.budapestopenaccessinitiative.org/> [Consulta: 25 abril 2016].

»Cabezas, Álvaro. 2007. Modelos de negocio en la publicación científica. En EC3noticias. <http://ec3noticias.blogspot.com/2007/01/modelos-de-negocioen-la-publicacin.html> [Consulta: 22 julio 2016].

»COAR-UNESCO. 2016. Joint statement about open access by COAR and UNESCO. $<$ https://www.coar-repositories.org/news-media/coar-and-unesco-joint-statement-about-open-access> [Consulta: 23 mayo 2016].

»CODESRIA. 2016. Dakar Declaration on Open Access Publishing in Africa and the Global South. <http://www.codesria.org/spip.php?article2595\&lang=en> [Consulta: 23 mayo 2016].

" COLCIENCIAS. 2015. COLCIENCIAS. Acceso a recursos bibliográficos e índices especializados <http://www.colciencias.gov.co/programa_estrategia/accesorecursos-bibliogr-ficos-e-indices-especializados> [Consulta: 16 setiembre 2015].

"CONACYT. 2015. CONACYT. <http://www.conacyt.gob.mx/conricyt/index.php/ conricyt/acerca-delconsorcio> [Consulta: 19 setiembre 2015].

»CRUE. 2016. CRUE. Universidades Españolas. <http://www.rebiun.org/publicaciones/Paginas/Anuarios- Estad\%25C3\%25ADsricos.aspx> [Consulta: 23 febrero 2016].

"Dosdoce. 2015. Evolución de los nuevos modelos de negocio en la era digital. Un estudio elaborado por dosdoce.com con el patrocinio de la plataforma con licencia de CEDRO (Centro Español de Derechos Reprográficos) <http://www.dosdoce. com/evolucion_nuevos_modelos_negocio_en_la_era_dgital_v2.pdf> [Consulta: 23 julio 2018].

»Eisen, Michael y Leslie B. Volsshall. 2016. Acoplando pre-prints y arbitrajes post publicación para una publicación científica económica, justa y eficaz [Publicado originalmente en el blog "it is not junk" de Michael Eisen]. En SciELO en Perspectiva. <https://blog.scielo.org/es/2016/o2/11/acoplando-pre-prints-yarbitrajes-post-publicacion-para-una-publicacion-cientifica-economica-justay-eficaz-publicado-originalmente-en-el-blog-it-is-not-junk-de-michael-eisen/> [Consulta: 23 julio 2018]. 
»Esteban, Pablo. 2018. El negocio de las revistas científicas. Viviana Martinovich analiza el mapa editorial de la ciencia internacional. En Página 12. Ciencia. 4 de abril. <https://www.pagina12.com.ar/105759-el-negocio-de-las-revistas-cientificas> [Consulta: 23 julio 2018].

»France Prefers to Pay (twice) for Papers by Its Researchers. 2014. En Open Knowledge International Blog. 11 de noviembre 2014. <https://blog.okfn. org/2014/11/11/france-prefers-to-pay-twice-for-papers-by-its-researchers/> [Consulta: 23 febrero 2016].

» Hahn, Trudi Bellardo. 1996. Pioners of the Online age. En Information Processing \& Management. Vol. 32, no.1, 33-48.

» Hartley, R. J.; E. M. Keen; J. A. Large y L. A. Tedd. 1990. Online searching: principles and practice. Londres: Bowker Saur.

»IFLA. 2001. IFLA Library Consortiun Preconference. IFLA. Boston: Information Technology and Libraries.

》IFLA. (2001). IFLA Library Consortiun Preconference. IFLA. Boston: Information Technology and Libraries. <http://origin-archive.ifla.org/IV/ifla67/pprog-e. htm> [Consulta: 23 julio 2018].

» Jisc. 2016. Academic journal markets, their limitations, and consequences for a transitions to open access: a thought piece. <https://www.jisc.ac.uk/reports/ academic-journal-markets-limitations-consequences-for-transition-to-openaccess> [Consulta: 11 julio 2016].

"Kolata, Gina. 2017. Many academics are eager to publish in worthless journals. En The New York Times. 30 de octubre. <https://www.nytimes.com/2017/10/30/ science/predatory-journals-academics.html> [Consulta: 23 julio 2018].

" Kopp, James J. 1998. Library consortia and information technology: the past, the present, the promise. En Information Technology and Libraries. Vol. 17, no. $1,7-12$.

"Lancaster, F. Wilfrid y Amy J. Warner. 1993. Information retrieval today. Arlington, Va: Information Resources Press.

" Larivière, Vincent; Stefanie Haustein y Philippe Mongeon. 2015. The oligopoly of academic publishers in the digital era. En PLoS ONE. Vol. 10, no. 6. https:// doi.org/10.1371/journal.pone.0127502

" Martín Gavilán, César. 2009. La industria editorial y el mercado de la información. Editores, agregadores, modelos de precio y licencias. En Temas de Biblioteconomía. <http://eprints.rclis.org/14237/1/industredit.pdf> [Consulta: 23 julio 2018].

» McFadden Allen, Barbara y Arnold Hirshon. 1998. Hanging together to avoid hanging separately: opportunities for academic libraries and consortia. En Information Technology and Libraries. Vol. 17, no. 1, 37-44.

"Neufeld, M. Lynne y Martha Cornog. 1986. Database history: from dinosaurs to compact disc. En Journal of the American Society for Information Science. Vol. 37, no. 4, 183- 190. https://doi.org/10.1002/(SICl)1097-4571(198607)37:4<183::AIDASI2>3.0.CO;2-W

" Owens, Simon. 2012. Is the Publishing Industry on the Verge of Disruption? Harvard complaints roil the debate over publishing and profits. En U.S. News. July 23, 2012. <https://www.usnews.com/news/articles/2012/o7/23/is-the-academic-publishing-industry-on-the-verge-of-disruption> [Consulta: 23 julio 2018]. 
»Peláez, J. 2016. Hackers de la ciencia. En El Español. <http://www.elespañol. com/ciencia/20160219/103489878_o.html> [Consulta: 23 febrero 2016].

"Potter, William Gray. 1997. Recent trends in statewide academic library consortia. En Library Trends. Vol. 45, no. 3, 416-434.

»Romanos de Tiratel, Susana. 2008. La revista científica: panorama internacional, latinoamericano y argentino En: Revistas argentinas de humanidades y ciencias sociales: visibilidad en bases de datos internacionales. Susana Romanos de Tiratel y colab. Buenos Aires: Instituto de Investigaciones Bibliotecológicas. Facultad de Filosofía y Letras. Universidad de Buenos Aires. p. 21-42

"Scalable Processor ARChitecture. 2003. Declaración de Bethesda sobre Publicación de Acceso Abierto. <http://www.senado.gob.mx/comisiones/ciencia_tecnologia/docs/accesoinfo_b3-3.pdf> [Consulta: 23 julio 2018].

"Schekman, Randy. 2013. How journals like Nature, Cell and Science are damaging science. En The Guardian. 9 de diciembre. <http://www.theguardian.com/ commentisfree/2013/dec/o9/how-journals-nature-science-cell-damage-science> [Consulta: 23 julio 2018].

》Schmitt, Janson. 2015. Can't disrupt the: Elsevier and the 25.2 Billion Dollar a Year Academic Publishing Business. <https://medium.com/@jasonschmitt/can-tdisrupt-this-elsevier-and-the-25-2-billion-dollar-a-year-academic-publishingbusiness-aa3b9618d40a> [Consulta: 5 abril 2015].

"Sociedad Max Planck. 2003. Declaración de Berlín sobre abierto. En GeoTrópico. Vol. 1, no. 2, 152-154. <https://openaccess.mpg.de/67627/Berlin_sp.pdf> [Consulta: 23 julio 2018].

»Stiglitz, Joseph E. y Bruce C. Greenwald. 2014. Creating a learning society: a new approach to growth, development, and social progress. New York: Columbia University Press.

" Tenopir, Carol. 2003. Information metrics and user studies. En Aslib Proceedings. Vol. 55, no. 1/2, 13-17. https://doi.org/10.1108/o0012530310462661

»Térmens-Gruells, M. 2007. Las revistas electrónicas en las universidades catalanas. En Consorcio de Bibliotecas de Universidades Catalanas COBUC. Vol. 1, no. 3, 104.

"Watt, Andrew. 2016. The indefensible price of accessing the giant's shoulders. $<$ http://mrandrewdwatt.com/the-indefensible-price-of-accessing-the-giantsshoulders/> [Consulta: 19 febrero 2016]. 